\title{
Um Sistema de Recomendação de Estratégias de Aprendizagem Baseado no Perfil de Motivação do Aluno: SisREA
}

\author{
Geovana de Souza Amaral ${ }^{1}$, David Brito Ramos ${ }^{1,2}$, Ilmara M. M. Ramos ${ }^{1,2}$, \\ Elaine H. T. de Oliveira ${ }^{1}$ \\ ${ }^{1}$ Instituto de Computação - Universidade Federal do Amazonas (UFAM) \\ Manaus -AM - Brasil \\ ${ }^{2}$ Instituto de Computação - Instituto Federal de Educação, Ciência e Tecnologia \\ do Amazonas (IFAM) - Campus Parintins - Parintins - AM - Brasil \\ \{david.ramos, ilmaramonteverde, elaine\} a icomp. ufam.edu.br
}

gsamaral05@gmail.com

\begin{abstract}
This work aims to present a learning recommendation system based on student motivation in a virtual learning environment. The data collection system uses the EMAPRE-U questionnaire, applied in an online judge system, and generates a student's motivation profile, from which it can recommend a learning strategy manually or automatically. As an initial validation of the results, the system recommended orienting 304 undergraduate students, of which 53 evaluated the goals received, since the pandemic paralyzed classes in 2020.
\end{abstract}

Resumo. Este trabalho tem como objetivo apresentar um sistema de recomendação de estratégias de aprendizagem baseado na motivação do aluno em um ambiente virtual de aprendizagem. O sistema de recomendação coleta dados utilizando o questionário EMAPRE-U, aplicado em um sistema de juiz online, e gera um perfil de motivação do aluno, a partir disso ele consegue recomendar uma estratégia de aprendizagem de forma manual ou automática. Como validação inicial dos resultados, o sistema recomendou estratégias a 304 alunos de graduação, dos quais 53 avaliaram as estratégias recebidas, uma vez que a pandemia paralisou as aulas em 2020.

\section{Introdução}

Com o aumento da utilização de ambientes virtuais de aprendizagem (AVAs) nos últimos anos, novos recursos têm sido oferecidos para dar suporte ao trabalho do professor nesse novo ambiente educacional. Dentre vários recursos que o AVA pode conter, têm-se os sistemas de recomendação. Os sistemas de recomendação tem como principal objetivo prover ao aluno itens de sugestão como aulas, materiais, estratégias de aprendizagem, entre outros, que sejam relevantes para o aluno e também adequados ao seu perfil [Vargas et al. 2017].

Os sistemas de recomendação em AVAs atuam como filtros de informação que sugerem o apoio educacional aos alunos diante de suas necessidades de aprendizado. Essa filtragem é possível, pois os sistemas utilizam o conhecimento dos dados dos alunos 
obtidos no AVA e na medida que mais informações são fornecidas melhor o sistema irá trabalhar na recomendação variando de perfil para perfil [Campos et al. 2017].

As estratégias de aprendizagem (EA) podem ser itens de recomendação em AVAs, e podem ser utilizadas para que o aluno seja favorecido no processo de educação atingindo seu principal objetivo, que é o aprendizado. Um dos meios para definir as estratégias de aprendizagem adequadas aos discentes, é conhecer o seu perfil motivacional baseado na teoria de metas de realização advinda da Psicologia Educacional [Ramos et al. 2020].

Segundo autores como [Badia e Monereo 2010, Veiga Simão 2004], é necessário investir em ações didáticas que auxiliem na forma de aprender, a pensar e a estudar do aluno. Dessa forma, fatores como a busca por ideais principais, processamento da informação, organização do tempo, e ambiente de estudo e regulação do esforço empregado pelo estudante, entre outros, têm sido foco de investigação de pesquisas psicoeducacionais, seja em meios de ensino presencial ou on-line.

As metas de realização são estabelecidas a partir de crenças constituídas por aspectos cognitivos e afetivos, expressas por meio de três diferentes tipos de orientações motivacionais, que são: a meta aprender, a meta performance-aproximação e a meta performance-evitação [Bzuneck e Boruchovitch 2016, Duffy e Azevedo 2015, Zenorini e Santos 2010].

Diante do exposto, o presente trabalho tem como objetivo apresentar um sistema de recomendação de estratégias de aprendizagem baseado na motivação dos alunos. Para isso, utilizou-se um instrumento de medição validado denominado Escala de Motivação para Aprendizagem de Universitários - EMAPRE-U [Santos et al. 2013, Zenorini e Santos 2010]. Com a classificação da motivação do aluno realizada, o professor pode utilizar o sistema para indicar uma sugestão de EAs a seus alunos.

O sistema proposto foi aplicado para 304 alunos de diferentes áreas de graduação. Desse total, apenas 53 alunos responderam à avaliação enviada sobre as estratégias recomendadas, uma vez que a pandemia paralisou as aulas em 2020.

\section{Trabalhos Relacionados}

O trabalho apresentado por [Vargas et al. 2017] descreve um sistema de recomendação baseado em um modelo cognitivo de aprendizagem através da análise do histórico dentro de uma ferramenta online de problemas de programação.O sistema utiliza uma técnica de ranking em jogos denominada ELO [Elo 1978]. A plataforma onde o mecanismo foi aplicado funciona através de um sistema de submissão, onde alunos enviam soluções algorítmicas para os problemas apresentados e recebem feedback automático.

A pesquisa de [Bezerra et al. 2017] consiste em um sistema de recomendação ubíquo integrando hipermídias baseadas em ontologias e tem como principal objetivo absorver as características do perfil do aprendiz e recomendar conteúdos de vídeo e texto localizados semanticamente do YouTube e Wikipédia. A arquitetura é composta por quatro webservices que executam todo o processo de obtenção das informações dos conteúdos, técnicas de recomendação e associação entre conteúdo e usuário.

O trabalho proposto por [Rezende et al. 2015] mostra um sistema de recomendação na área educacional que tem por objetivo acompanhar dados do aluno quando esse interage com aplicações educacionais, para que seja possível recomendar 
objetos de aprendizagem adequados às suas preferências. É utilizada uma ontologia denominada PERSONNA, que significa Proposta de Ontologia de Contexto e Perfil de Aluno para Recomendação de Objetos de Aprendizagem. Essa ontologia filtra as informações pertinentes do aluno de acordo com o contexto de localização e facilita o processo de recomendação de objetos de aprendizagem.

\section{Metodologia}

Como metodologia do sistema, primeiramente foram identificadas as motivações dos alunos no AVA sob a ótica da Teoria de Metas de Realização conforme proposto por [Ramos et al. 2020] e para isso utilizou-se um instrumento de medição validado denominado EMAPRE-U [Santos et al. 2013, Zenorini e Santos 2010]. O questionário foi aplicado em um sistema de juiz online denominado CodeBench ${ }^{1}$. Foi utilizado um conjunto de sugestões de EAs associadas aos modelos de comportamento levantado por [Ramos 2020]. A associação de sugestões serve para auxiliar o estudante no uso de EAs mais adequadas ao seu perfil.

Em seguida, foi desenvolvida uma ferramenta integrando o modelo de comportamento do aluno às EAs, onde as recomendações de estratégias puderam ser realizadas e avaliadas. O sistema foi desenvolvido para Web devido à mobilidade e portabilidade proporcionada por este tipo de arquitetura. Foi utilizada a linguagem de programação PHP $^{2}$, que é uma linguagem de script open source de uso geral, e utilizada principalmente para o desenvolvimento web. Para o banco de dados, foi utilizado o $\mathrm{MySql}^{3}$, que é um sistema de gerenciamento de banco de dados que utiliza a linguagem SQL como interface.

Após o desenvolvimento do sistema, foi realizada uma avaliação das EAs enviadas aos alunos de graduação que utilizaram o ambiente nos semestres de 2019/2 e 2020/1. As avaliações dos estudantes ficam armazenadas no banco de dados para que os dados possam ser analisados posteriormente.

Em um trabalho anterior [Ramos et al. 2020], as estratégias de aprendizagem foram selecionadas e então associadas a cada tipo de meta. Dessa forma, já se sabe previamente as estratégias de aprendizagem adequadas a cada tipo de meta dos alunos.

\section{O SisREA}

\subsection{Arquitetura}

A arquitetura do sistema proposto denominado Sistema de Recomendação de Estratégias de Aprendizagem - SisRea - está apresentada na Figura 1. Primeiramente, são coletados os dados obtidos via questionário que são utilizados para atribuir a motivação do estudante e compõem o módulo do estudante. Em seguida, há o módulo de classificação da motivação do aluno, onde é calculada a motivação de acordo com a teoria adotada e atribuído o resultado ao modelo do estudante. Depois, têm-se o módulo de recomendação que utiliza os dados sobre a motivação dos estudantes aliados às EAs registradas no sistema.

\footnotetext{
${ }^{1}$ Disponível em: www.codebench.icomp.ufam.edu.br

${ }^{2}$ Disponível em: www.php.net

${ }^{3}$ Disponível em: www.mysql.com
} 
As EAs sempre estarão associadas às motivações. O professor, através de uma interface, é capaz de visualizar as motivações e as estratégias disponíveis para cada tipo de meta, e dessa maneira pode sugerir aos alunos pelo próprio sistema. As estratégias são enviadas via e-mail, e torna-se disponível para a avaliação através de uma escala de avaliação representada por estrelas. Com isso, é verificado se a estratégia recomendada é significativa para o estudante, e em paralelo, é avaliado se de fato ela está correlacionada com a sua motivação.

\section{Figura 1. Arquitetura do SisREA}

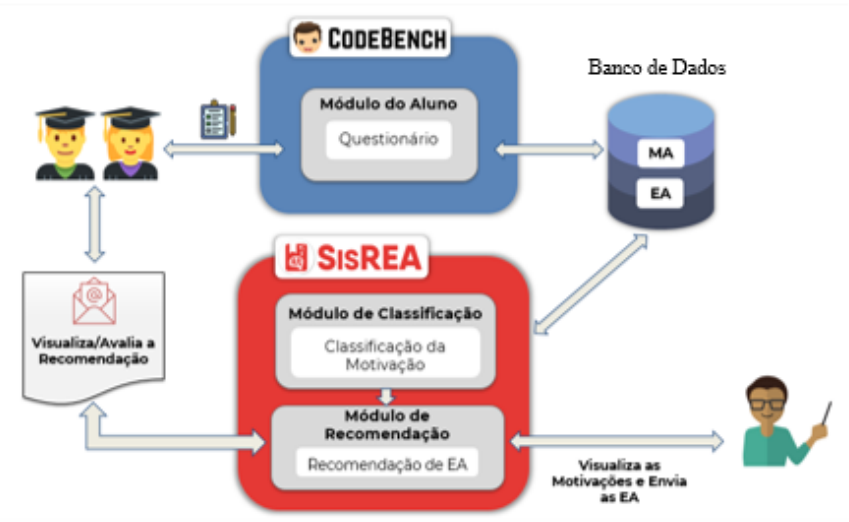

\subsection{Módulo do Aluno}

Nesse módulo da arquitetura, como mostrado na Figura 1, o aluno ao utilizar o AVA recebe um questionário, como instrumento de medição, denominado EMAPRE-U. Para [Santos e Mognon 2016], é indispensável a utilização de instrumentos de medida adequados aos domínios específicos de interesse e com boas qualidades psicométricas. A escala de motivação, no âmbito educacional, é um meio desenvolvido na área da psicologia educacional que é testado e validado para mostrar as suas propriedades psicométricas [Ramos et al. 2020]. Após responder o questionário no AVA, as informações dos alunos ficam salvas no banco de dados e depois utilizadas no módulo de classificação.

\subsection{Módulo de Classificação}

O módulo de classificação tem como objetivo coletar os dados dos alunos do questionário EMAPRE-U e classificá-los de acordo com a escala de motivação no sistema de aprendizagem. As motivações dos alunos (MA) são definidas de acordo com a Teoria de Metas de Realização e estão armazenadas no banco de dados, como pode ser visto na Figura 1. A EMAPRE-U foi desenvolvida por [Santos et al. 2013, Zenorini e Santos 2010] e possui 28 itens constituídos com três fatores classificados. O primeiro é a meta aprender com 12 itens referentes ao esforço e a busca de novos conhecimentos pelo estudante. O segundo fator agrupa nove itens da meta performance-aproximação caracterizados pela preocupação do aluno em se destacar em relação aos colegas. Por fim, a meta performance-evitação, com sete itens concernentes ao cuidado do aluno em não demonstrar incapacidade diante dos outros. 


\subsubsection{Perfis das Metas Realização}

Os alunos com meta de domínio (mastery) ou de aprender (learning) se esforçam para aumentar a sua competência. Alunos com este perfil buscam aprender e desenvolver suas habilidades, eles focam mais no seu progresso pessoal do que tentar ser melhor que os outros [Romero et al. 2020]. As pesquisas também têm buscado a relação da orientação de metas com outros fatores. Por exemplo, a meta aprender está positivamente relacionada com extroversão, abertura, persistência, conscienciosidade e afeto positivo, e inversamente relacionada com neuroticismo, ansiedade e raiva [Romero et al. 2020]. Pessoas com este perfil tendem a ser organizadas e persistentes diante das dificuldades.

Alunos cuja meta é performance-aproximação buscam demonstrar mais competência em relação a outros alunos. Eles também querem ganhar vantagens, obter altas pontuações e alcançar os objetivos o mais rápido possível, sem necessariamente obter conhecimento ou desenvolver habilidades para isso [Romero et al. 2020]. Alunos com a meta orientada a performance, tendem a utilizar estratégias de aprendizagem mais rasas, como atalhos e macetes, se comparados com alunos orientados a aprendizagem [Romero et al. 2020]. Eles, por exemplo, respondem melhor aos emblemas de conquistas como feedback, pois é um indicador para alto desempenho [Auvinen et al. 2015].

Alunos com a meta performance-evitação, buscam evitar a demonstração de incompetência. [Auvinen et al. 2015]. Eles também buscam não passar por situações, que para eles seja considerado como constrangedor. Alunos com a meta performance-evitação apreciam mais os mapas de calor, pois estes mostram o aluno um mapa que estima resultados ruins [Auvinen et al. 2015]. Metas orientadas a performance estão positivamente relacionadas a neuroticismo, raiva, ansiedade e vergonha. Esse perfil é inversamente relacionado com persistência em tarefas, abertura, extroversão [Romero et al. 2020].

\subsection{Módulo de Recomendação}

O módulo de recomendação utiliza os dados sobre a motivação do estudante, mais as EAs armazenadas no banco de dados. O docente, através de uma interface, visualiza as motivações e as estratégias disponíveis para cada tipo de motivação, e envia as recomendações aos estudantes. De acordo com um mapeamento de soluções tecnológicas em sistemas de recomendação realizado por [Campos et al. 2017], é possível notar que a maior parte das soluções utilizadas na modalidade de educação a distância fazem uso de técnicas de filtragem de conteúdo, colaborativa e híbrida. Neste trabalho, utilizou-se a técnica de recomendação baseada em filtragem colaborativa que tem como principal vantagem criar a possibilidade de recomendar itens automaticamente, uma vez que são recomendados os itens considerados mais relevantes por usuários com motivações semelhantes. Os algoritmos de filtragem colaborativa funcionam pesquisando um grande grupo de usuários ou itens e encontrando uma lista menor com gostos semelhantes aos seus. No sistema proposto, primeiramente o docente visualiza as motivações e as estratégias disponíveis para cada tipo de meta, e as sugere aos estudantes pelo próprio sistema, após isso, os alunos recebem as EAs e podem classificar através de escala de avaliação que varia de 1 a 5 .

Nessa abordagem, essas avaliações permitem descobrir médias para as EAs, com isso o Sistema de Recomendação pode descobrir padrões de comportamento e recomendar automaticamente os itens considerados mais relevantes pelos usuários com interes- 
ses semelhantes. Caso as informações não sejam suficientes para criar uma base de recomendação de EAs, é sugerida a motivação mais avaliada dentro do perfil de motivação do estudante.

\section{Resultados da Aplicação}

O SisREA foi aplicado em um AVA denominado CodeBench, que é um sistema no formato de juiz online com o intuito de auxiliar os alunos sobre conceitos de programação. Através do CodeBench os alunos responderam ao EMAPRE-U, após isso as respostas dos estudantes foram armazenadas no banco de dados do sistema.

O módulo de classificação do SisREA acessa as respostas e calcula a orientação de meta do estudante. Com isso, a meta com o maior índice calculado é lançada ao perfil do aluno.Com a classificação realizada, o professor pode utilizar o SisREA para indicar uma sugestão de EAs a seus alunos. Durante a validação do sistema, foram coletadas as respostas dos questionários de alguns estudantes voluntários, de cursos de diversas áreas, os quais utilizaram o ambiente nos semestres de 2019/2 (152 estudantes) e 2020/1 (152 estudantes), somando 304 estudantes. Desse total, apenas 53 alunos avaliaram as estratégias recebidas por email, uma vez que a pandemia paralisou as aulas em 2020.

Para avaliação das etratégias recomendadas utilizou-se a escala de Likert de 5 pontos com valores variando de 'concordo totalmente' (5) até 'discordo totalmente' (1). Do total de alunos, apenas 53 alunos avaliaram as estratégias recebidas por email, uma vez que a pandemia paralisou as aulas em 2020.

Com a obtenção das respostas dos alunos, foi possível observar que o sistema conseguiu realizar as recomendações e também coletar as notas dadas pelos alunos sobre as EAs durante a realização da validação.

No gráfico da Figura 2 são exibidas as notas coletadas. Percebe-se que o a maior porcentagem da avaliação dos alunos está na classificação em 5 estrelas com $68 \%$, em seguida $9 \%$ para 4 e 3 estrelas, $4 \%$ duas estrelas e por fim $10 \% 1$ estrela. Os resultados mostram-se promissores e os alunos se identificaram com as estratégias recomendadas. Já as menores porcentagens apresentadas, representam que algumas estratégias não se adequaram aos alunos e pode indicar uma reformulação ou até mesmo a retirada delas do sistema.

Figura 2. Gráfico das avaliações das recomendações

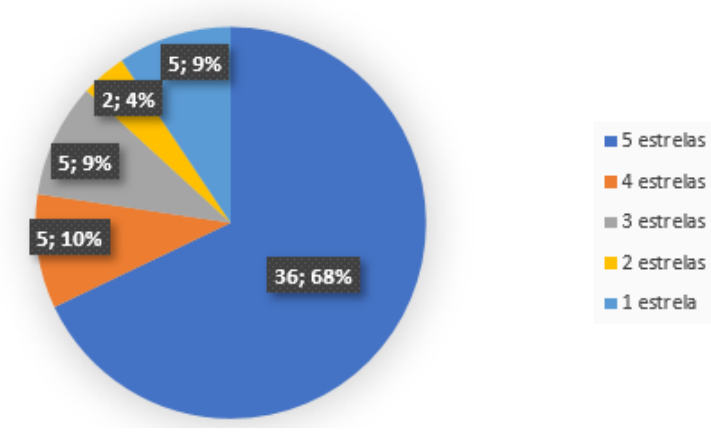




\subsection{Interface Professor/Tutor}

$\mathrm{Na}$ interface do professor/tutor pode-se visualizar, as motivações dos estudantes classificados, as estratégias registradas no sistema e as opções de recomendação de EAs. O docente também pode visualizar as estratégias recomendadas para cada estudante, assim como as notas dadas por eles, caso já as tenham avaliado.

Na Figura 3, têm-se a primeira página ao logar no sistema, nela são encontradas as turmas cadastradas no CodeBench. Também é possível visualizar o curso, o ano e o semestre vigente.

Figura 3. Tela Inicial do SisREA

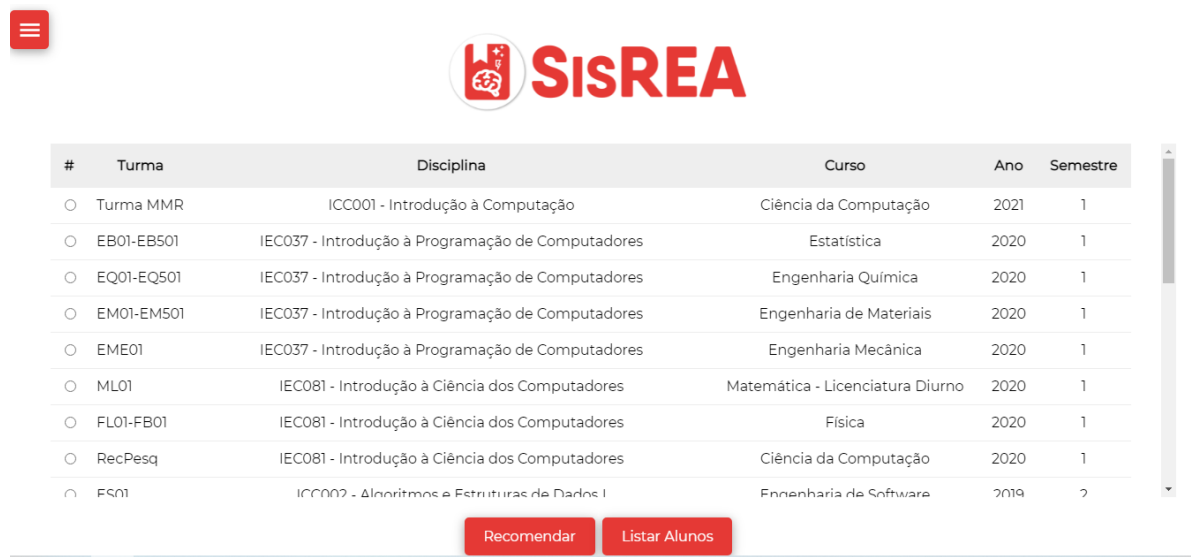

A tela de recomendação de estratégias está apresentada na Figura 4. Nessa tela, o professor pode visualizar os nomes dos alunos, o seu id, a motivação atribuída, bem como os valores calculados em cada nível de motivação. As estratégias estão dentro de uma lista de seleção, onde o professor pode selecionar qual será enviada ao aluno.

Figura 4. Tela Recomendação das Estratégias

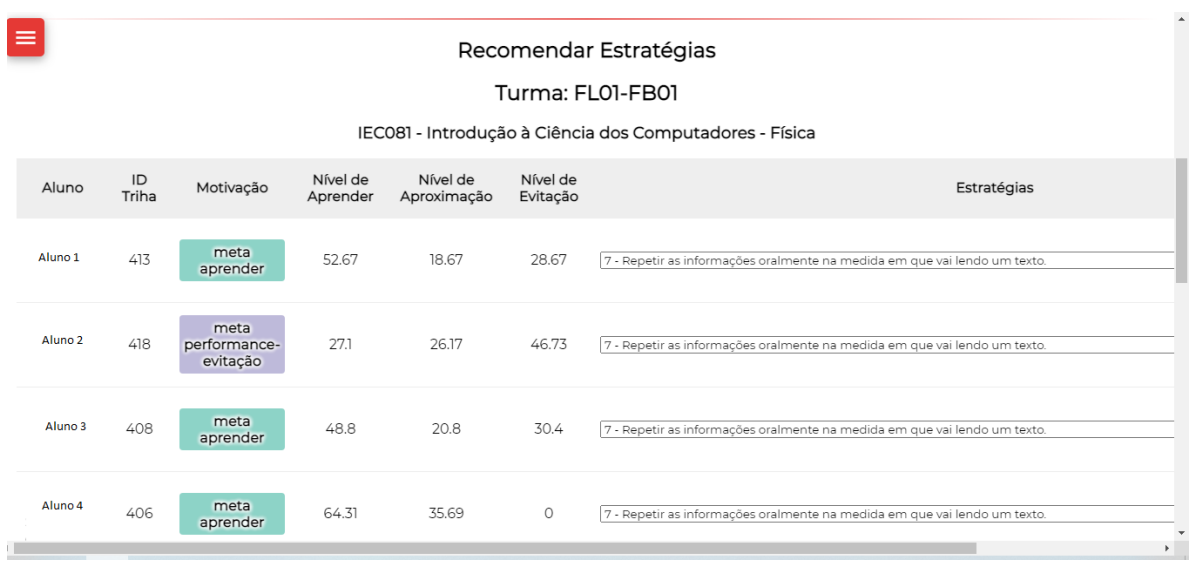

\subsection{Visualização da Recomendação}

Os alunos recebem a recomendação através do e-mail cadastrado no AVA, onde é exibida a EA e uma classificação na escala de Likert, conforme a Figura 5. As avaliações dos estudantes são armazenadas em banco de dados e podem ser utilizadas para identificar 
Figura 5. Visualização das Estratégias

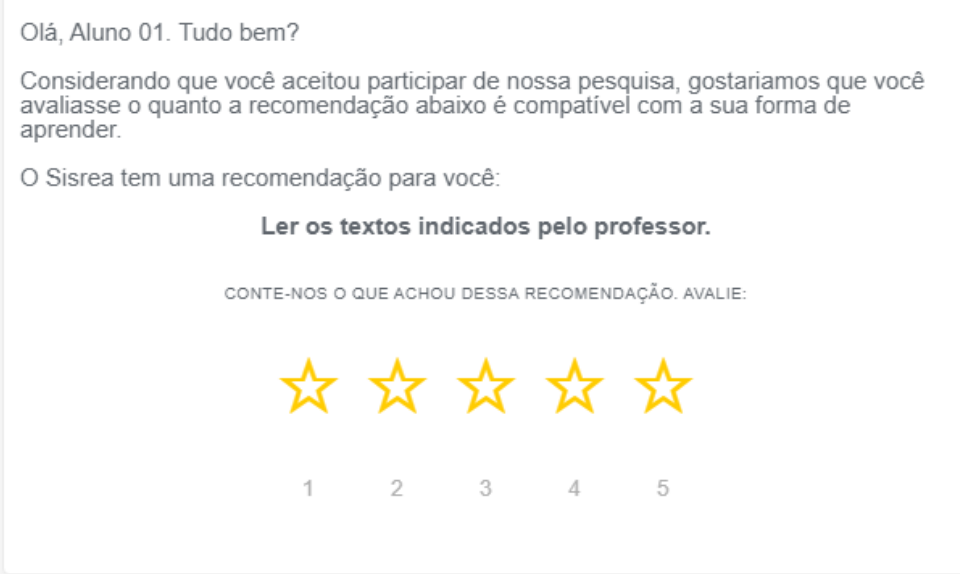

quais foram as melhores estratégias sugeridas e por consequência tornar cada vez melhor o sistema de recomendação.

Essa etapa de avaliação dos alunos contribui para que o professor tenha um parecer da opinião dos alunos em relação às estratégias enviadas por ele. Além disso, as notas dadas pelos alunos são salvas no banco de dados e alimentam o algoritmo de recomendação. $\mathrm{O}$ algoritmo de recomendação processa as notas das EAs e com isso pode ser utilizado para sugerir de forma automática quais serão as estratégias mais relevantes.

\section{Considerações Finais}

Os sistemas de recomendação são recursos que sugerem itens para os usuários de forma automatizada e personalizada, sem a necessidade do usuário formular uma consulta para encontrar os itens do seu interesse. No âmbito educacional, esses sistemas são explorados em Ambientes Virtuais de Aprendizagem e possuem o intuito de dar suporte ao trabalho do professor/tutor no processo educacional. Esse trabalho teve como objetivo principal propor um sistema de recomendação de estratégias de aprendizagem baseado no perfil motivacional do aluno. Para alcançar os objetivos desse trabalho, primeiramente fez-se um levantamento bibliográfico sobre sistemas de recomendação em ambientes virtuais.Em seguida, o sistema proposto foi aplicado para alunos de diversas áreas do conhecimento em disciplinas de programação, dentro do ambiente CodeBench no segundo semestre de 2019 até o primeiro semestre de 2020.

Depois, foram coletadas as respostas dos alunos através do questionário EMAPRE-U. Após isso, o SisREA coletou essas respostas do banco de dados e fez a classificação da motivação baseado na teoria de metas de realização. A partir disso, o professor tutor pode conhecer o perfil de motivação do aluno e dessa forma escolher uma estratégia de aprendizagem apropriada para cada aluno, a qual é enviada ao e-mail individual dos alunos. Após isso, há a avaliação dos alunos em relação às estratégias recebidas, dessa forma as notas atribuídas alimentam o sistema de recomendação para poder sugerir estratégias relevantes.

Por fim, foi alcançada a recomendação de estratégias de aprendizagem de forma manual ao alunos, no entanto não foi possível testar as recomendações automáticas feitas 
a partir da utilização da técnica de recomendação de filtragem colaborativa.

Como trabalhos futuros, propõe-se testar as recomendações automáticas desenvolvidas no SisREA. Realizar um estudo mais aprofundado com relação aos algoritmos de recomendação e realizar experimentos mais elaborados para definir a melhor abordagem de recomendação. Outra proposta, é aliar o perfil de motivação dos alunos com dados implícitos do ambiente virtual de aprendizagem para aprimorar a recomendação automática.

\section{Referências}

Auvinen, T., Hakulinen, L., e Malmi, L. (2015). Increasing students' awareness of their behavior in online learning environments with visualizations and achievement badges. IEEE Transactions on Learning Technologies, 8(3):261-273.

Badia, A. e Monereo, C. (2010). Ensino e aprendizagem de estratégias de aprendizagem em ambientes virtuais. Psicologia da Educação virtual-Aprender e ensinar com as tecnologias da informação e da comunicação, pages 311-328.

Bezerra, S. F., Silva, S., Neto, F. M., Silva, P., e de Sousa Monteiro, B. (2017). Sistema de recomendação ubíquo integrando hipermídias baseada em ontologia. In Brazilian Symposium on Computers in Education (Simpósio Brasileiro de Informática na Educação-SBIE), volume 28, page 1793.

Bzuneck, J. A. e Boruchovitch, E. (2016). Motivação e autorregulação da motivação no contexto educativo. Psicologia Ensino \& Formação, 7(2):73-84.

Campos, A. d., Hollerweger, L., Santos, G., Farias, A. F., e Behar, P. A. (2017). Mapeamento de soluções tecnológicas em sistemas de recomendação educacionais em âmbito brasileiro. Informática na educação: teoria \& prática. Porto Alegre. Vol. 20, n. 3 (set./dez. 2017), p. 78-93.

Duffy, M. C. e Azevedo, R. (2015). Motivation matters: Interactions between achievement goals and agent scaffolding for self-regulated learning within an intelligent tutoring system. Computers in Human Behavior, 52:338-348.

Elo, A. E. (1978). The Rating of Chessplayers, Past e Present. Arco Pub., New York.

Ramos, D., Ramos, I., Gasparini, I., e Oliveira, E. (2020). Um framework conceitual para recomendação de estratégias de aprendizagem utilizando motivação e trilhas de aprendizagem. In Anais do XXXI Simpósio Brasileiro de Informática na Educação, pages 872-881, Porto Alegre, RS, Brasil. SBC.

Ramos, D. B. (2020). Motivação e Estratégias de Aprendizagem. http://mobmoodle.icomp.ufam.edu.br/projeto/?page_id=159.

Rezende, P. A. A., Pereira, C. K., Campos, F., David, J. M. N., e Braga, R. (2015). Personna: proposta de ontologia de contexto e perfil de alunos para recomendação de objetos de aprendizagem. Revista Brasileira de Informática na Educação, 23(01):70.

Romero, M., Hernández, J. M., Juola, J. F., Casadevante, C., e Santacreu, J. (2020). Goal orientation test: An objective behavioral test. Psychological reports, 123(4):14251451. 
Santos, A. A. A. d., Alcará, A. R., e Zenorini, R. d. P. C. (2013). Estudos psicométricos da escala de motivação para a aprendizagem de universitários. Fractal: Revista de Psicologia, 25:531-546.

Santos, A. A. A. d. e Mognon, J. F. (2016). Motivation assessment scale for learning in higher education (emapre-u): validity evidence. Psico-USF, 21:101-110.

Vargas, A. P., Santos, R., da Costa Botelho, S. S., Tonin, N., e Bez, J. (2017). Um sistema de recomendação baseado em um modelo cognitivo de aprendizagem. In $\mathrm{Bra}$ zilian Symposium on Computers in Education (Simpósio Brasileiro de Informática na Educação-SBIE), volume 28, page 1667.

Veiga Simão, A. M. (2004). Integrar os princípios da aprendizagem estratégica no processo formativo dos professores, pages 95-106.

Zenorini, R. d. P. C. e Santos, A. A. A. (2010). Escala de metas de realização como medida da motivação para aprendizagem. Revista Interamericana de Psicología/Interamerican Journal of Psychology, 44(2):291-298. 